\title{
Upper Limb Neural Tissue Extensibility in Apparently Asymptomatic Professional Computer users
}

\author{
Shahanawaz SD \\ Assistant Professor, Dr. D.Y.Patil College of Physiotherapy,India \\ shanu.neuropt@gmail.com
}

\begin{abstract}
:
Introduction: Assessment of peripheral nerve disorders can be classified into clinical tests such nerve conduction test, performed to examine nerve functions and Neural Tissue Tension Tests designed to find out increased mechanosensitivity of the nerve being tested. Upper Limb Neural Tissue Tension Tests (ULTT) is a good tool for assessment of early affection of the neural tissue.

Purpose of Study: Maintenance of prolonged posture with relatively similar upper extremity activities provoke reduced mobility in Nerve tissue. Presence of abnormal neural tension in asymptomatic population may be an early marker of impending radiculopathy during degenerative changes of the spine in the later stages of life. Identification of these symptoms and correction of the adverse tension in the early stages may serve as a prophylaxis for the later course of life.
\end{abstract}

Aim of study: To observe the extensibility of Nerve tissue mobility of upper limb peripheral nerves in professional computer users.

Methodology: ULTT were performed in the individuals of age 20-30 years using computers for at least about 8 hours per day on both dominant and non dominant hand. The sensitivity and type of symptoms provoked with the tests were observed and analyzed.

Conclusion: Abnormal posture leads to abnormal neural tissue mobility. Hence stretching the neural tissue at appropriate age may be precautionary for future problems.

Keywords: Peripheral Nerve, Nerve Tension, Nerve Glides, Posture, Upper Quadrant, Neural Tension Test.

\section{INTRODUCTION}

Nerve tissue all throughout the body is in some way or the other a continuation of the same myelin sheath. Myelin sheath is the covering of Nerve tissue. The pure mechanical interface may be a fascial sheet or a blood vessel, a more relevant interface such as muscle or ligament may be adjacent to it [1, $3]$.

The nerve fibres are packed within endoneurial connective tissue and bundled into fascicles. These fascicles are surrounded by the perineurium, a relatively thin but distinctive lamellate sheath of connective tissue composed of tightly packed collagenous and elastic fibres. The collagenous and elastic fibres are circularly, obliquely and longitudinally in the fascicles. Therefore, Peripheral nerves are relatively resistant to mechanical load. The perineurium is the main component giving tensile strength and elasticity to the nerve trunk $[2,3]$.

The perineurium surrounds the fascicles and permits some movement of the fibres inside the fascicle. The entire nerve trunk is surrounded by the mesoneurium with its extraneural gliding surface called adventitia that permits excursion of the nerve trunk during joint motion. This extraneural gliding surface together with the intraneural sliding of fascicles against each other in deeper layers, present the normal gliding mechanism during joint motion. Thus the relationship of nerve position to the axis of a joint helps to establish the effect of a movement on neural tissue [2, 3, and 4]. Under normal physiological conditions, imposed by posture and movement, nerves are exposed to various mechanical stresses. Tensile stress may be applied to tissues either parallel or perpendicular to the length of the nerve, causing respective longitudinal or transverse stress in the nerve. When a joint motion cause elongation of the nerve bed, the nerve is inherently placed under tensile stress and accommodates the stress by both elongating and gliding [7, 9].

Displacement or gliding of a nerve relative to the surrounding nerve bed is called "excursion." 
The anatomical relationship between the nerve and the axis of rotation of the moving joint determines the direction and magnitude of nerve excursion. The term "convergence" is used to describe the glide of nerve towards moving joint with elongation of nerve bed, while the term "divergence" is used when nerve bed is shortened and nerve glides away from the joint when the tension is relieved [4]. With limb movement, nerve excursion occurs first in the nerve segment immediately adjacent to the moving joint. As limb movement continues, excursion occurs at nerve segments that are progressively more distant from the moving joint. Similarly, the magnitude of excursion is greatest in the nerve segments adjacent to the moving joint and is least in the nerve segments distant from the joint. There are a number of factors that affect nerve compliance and thus dictate the level of strain, excursion, and transverse contraction in the nerve during limb movement $[3,4,9]$.

In addition to tensile stress, nerves are exposed statically and dynamically to compressive stresses. As the laws of physics dictate that as a nerve is elongated under tensile force, the nerve undergoes transverse contraction, which is resisted by the fluid and nerve tissue contained within the connective tissue sheath $[9,14]$.

In static posture, the body and its segments are aligned and maintained in certain positions. In an analysis of the muscular forces in sitting position it was described that erect active sitting required more energy as compared to the slouched forward bend relaxed sitting as the line of gravity passed anteriorly to the spine [6]. In this position the passive tension on the neural tissue is increased.

To evaluate the mechanosensitivity of the brachial plexus and the median, radial and ulnar nerve neurodynamic tests for the upper limb were designed. A test is considered positive if symptoms of pain and paresthesia can be reproduced and symptoms can be altered by structural differentiation. Structural differentiation such as alteration in movement at a joint away from the painful area, by further loading or unloading the nervous system. With joint movements the nervous system slides on the underlying nerve bed and is subject to subsequent compression and stretch although a healthy nervous system can tolerate this loading, very low levels of stretch and compression are well enough to make it symptomatic. The evaluation of the provocation tests of neural tissue are based on the increased mechanosensitivity of this structure. Upper limb tension test [ULTT] also known as Brachial plexus tension tests or neural tissue provocation tests [NTPT] are used as tool for assessment of neural tissue components for sliding and gliding $[6,7,8,9]$.

The base neural tension test for example Passive neck flexion (PNF), Straight legs raise (SLR), prone knee bend (PKB), Slump Test and Upper limb tension test (ULTT) are considered as Neurodynamic Tests $[6,7]$.

\section{OBJECTIVES OF THE STUDY}

The study was conducted to observe the extensibility of neural tissue in asymptomatically computer professional users with sitting posture maintained for minimum of 7-8 hours per day.

\section{Methodology}

60 right hand dominant individuals between the age 20 to 30years were selectedby the simple random sampling method and an informed consent was taken from each of the candidate before participation in the study. The individuals were professional computer users who preferred sitting for at least 7 to 8 hours per day for their work purpose and had asedentary lifestyle. They had been working with the computers from minimum of 2 years and presently did not complaint of any pain in around the upper quadrant of the body. Individuals with any complaints of shoulder and/ or cervical pain in recent past were not considered as the part of the study to avoid any difference in the study procedure. All the candidates were evaluated after the work timings according to their convenience.

All the neural tissue tension tests procedure was performed exactly in the same sequence as described by Butler D [7, 8].

The individuals were evaluated for all the three nerve biased neural tissue provocation tests [NTPT]. There are four tests each of which is biased towards a particular nerve [7].

ULTT1a- Median nerve

ULTT1b- Median nerve

ULTT2- radial nerve

ULTT3- Ulnar nerve 
For further sensitization of the nerve trunk various components can be added at the levels of different joints starting from cervical motions. Contralateral cervical lateral flexion increases the symptoms. Similarly, for desensitization the same joints are taken in the opposite range for reduction of symptoms. Ipsilateral cervical lateral flexion reduces the symptoms

A test is considered positive if one or more of the following are found.

All or part of the symptoms has been reproduced. Symptoms differ from the normal response are produced.

The range of movement in the symptomatic limb is different to that of the other limb.

Table1. ULTT 1: Median Nerve Bias

\begin{tabular}{|l|l|}
\hline JOINT & MOVEMENT \\
\hline Shoulder Girdle & Depression \\
\hline Shoulder Joint & Abduction \\
\hline Forearm & Supination \\
\hline Wrist And Finger & Extension \\
\hline Shoulder Joint & Lateral Rotation \\
\hline Elbow & Extension. \\
\hline
\end{tabular}

The sensitizing component for ULTT 1 is cervical lateral flexion away from the testing side, and the desensitizing test is lateral flexion towards the testing side.

Table2. ULTT 1b: Median Nerve Bias

\begin{tabular}{|l|l|}
\hline JOINT & MOVEMENT \\
\hline Shoulder Girdle & Depression \\
\hline Shoulder Joint & Abduction 10degree \\
\hline Elbow & Extension \\
\hline Arm & Lateral Rotation \\
\hline Forearm & Supination \\
\hline Wrist And Finger And Thumb & Extension \\
\hline
\end{tabular}

The sensitizing test is cervical lateral flexion from the testing side or shoulder abduction. The desensitizing test is the lateral flexion towards the testing side or release of the shoulder girdle depression.

Table3. ULTT 2: Radial Nerve Bias

\begin{tabular}{|l|l|}
\hline JOINT & MOVEMENT \\
\hline Shoulder Girdle & Depression \\
\hline Shoulder Joint & Abduction 10degree \\
\hline Elbow & Extension \\
\hline Arm & Medial Rotation \\
\hline Forearm & Pronation \\
\hline Wrist And Finger And Thumb & Flexion \\
\hline
\end{tabular}

The sensitizing test is cervical lateral flexion away from the testing side or shoulder abduction. Shoulder girdle protraction will sensitize the suprascapular nerve. The desensitizing test is lateral flexion towards the testing side or release of the shoulder girdle depression

Table4. ULTT 3: Ulnar Nerve Bias

\begin{tabular}{|l|l|}
\hline JOINT & MOVEMENT \\
\hline Wrist And Finger And Thumb & Extension \\
\hline Forearm & Pronation \\
\hline Elbow & Flexion \\
\hline Shoulder Girdle & Depression \\
\hline Shoulder Joint & Abduction \\
\hline Arm & Medial Rotation \\
\hline
\end{tabular}

The sensitizing test is cervical lateral flexion away from the testing side and flexion towards the symptomatic side to desensitize. Normal responses to the upper limb tension test have only been investigated for the ULTT 1 . The normal response for this test includes the following deep ache or stretch in the cubital fossa extending to the anterior and radial aspects of the forearm and handdefinite tingling in the thumb and first three fingers. 
A stretch feeling over the anterior aspect of the shoulder

\section{Results}

The data was analyzed using the unpaired $\mathrm{T}$ test. The readings of the all the three tests were compared for the same individual.

During this study, from figure 1,ULTT 1,we observed that, out of 60 subjects,

5 males in right side, 4 males in left side, 9 females in right side and 11 females in left side reported only stretch. 1 male in right as well as left side and 5females in right side and 3 in left side experienced only pain,strech plus pain was experienced by 8 males in right side, 10 males in left side, and 4 females in right side, 5 females in left side. 15 males in right side while 14 males in left side reported paresthesia in case of females 12 in right side and 11 in left side reported the same ,only one of the male did not experienced any one of the symptoms mentioned above.

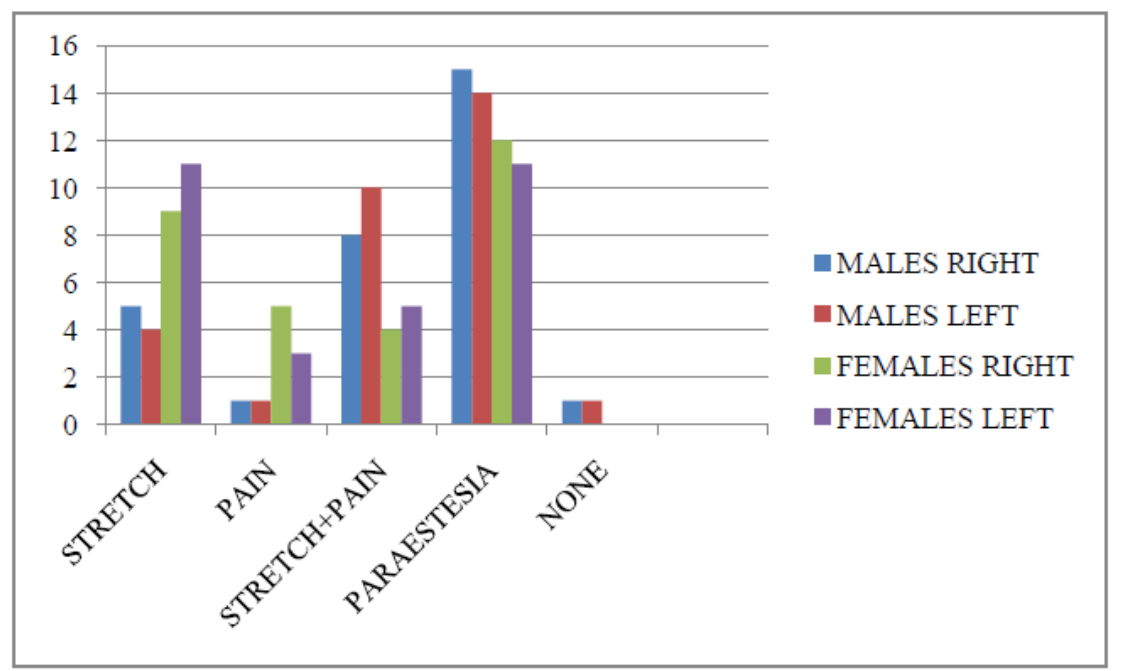

Figure1. Showing symptoms for ULTT 1 median nerve bias

Table1. ULTT 1 :median nerve bias

\begin{tabular}{|l|l|l|l|l|}
\hline \multirow{2}{*}{ Parameters } & \multicolumn{2}{|l|}{ Males } & Females & \\
\cline { 2 - 5 } & Right & Left & Right & Left \\
\hline Stretch & 5 & 4 & 9 & 11 \\
\hline Pain & 1 & 1 & 5 & 3 \\
\hline Stretch+Pain & 8 & 10 & 4 & 5 \\
\hline Paraestesia & 15 & 14 & 12 & 11 \\
\hline None & 1 & 1 & 0 & 0 \\
\hline
\end{tabular}

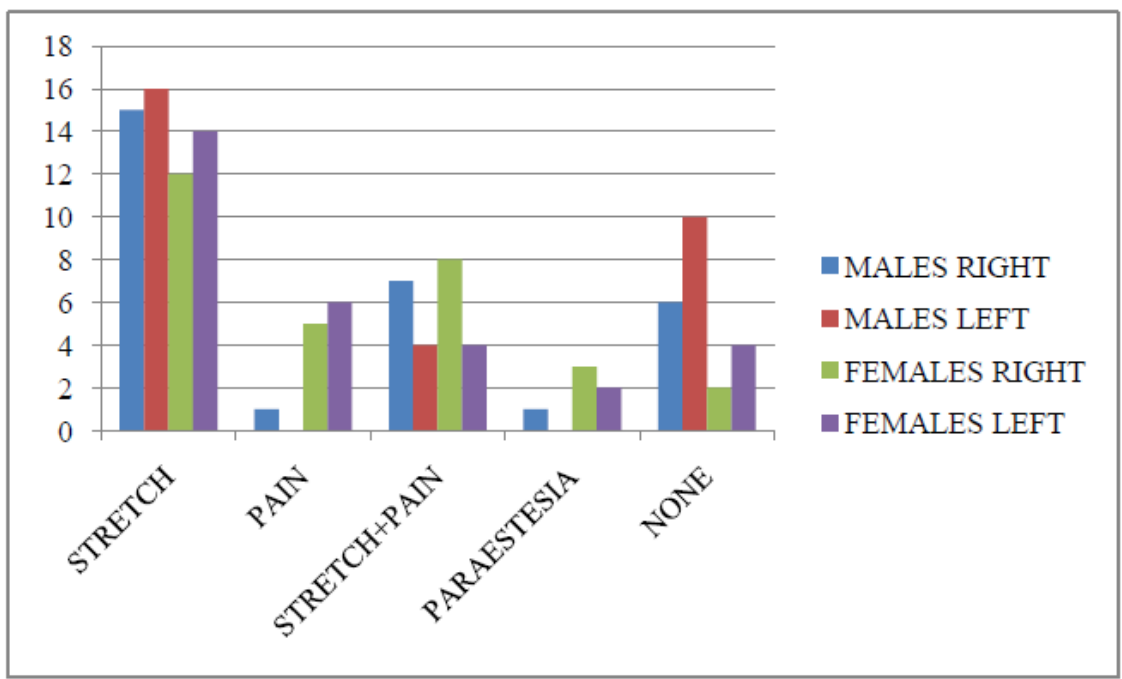

Figure2. Sowing results for ULTT 2 Radial nerve bias 
Upper Limb Neural Tissue Extensibility in Apparently Asymptomatic Professional Computer users

Table2. ULTT 2: Radial nerve bias

\begin{tabular}{|l|l|l|l|l|}
\hline \multirow{2}{*}{ Parameters } & Males & Females & \multicolumn{2}{l|}{} \\
\cline { 2 - 5 } & Right & Left & Right & Left \\
\hline Stretch & 15 & 16 & 12 & 14 \\
\hline Pain & 1 & 0 & 5 & 6 \\
\hline Stretch+Pain & 7 & 4 & 8 & 4 \\
\hline Paraestesia & 1 & 0 & 3 & 2 \\
\hline None & 6 & 10 & 2 & 4 \\
\hline
\end{tabular}

From figure 3, ULTT 2,we observed,

Out of 60 subjects, stretch was experienced by 15 males in right side, 16 males in left side, 12 females in right side and 14 females in left side.only 1 male candidate reported pain in right side while 5 females in right side and 6 females in left side reported the same.

7 males in right side, 4 in left side, 8 females in right side reported stretch plus pain,in case of paresthesia 1 male in rught side, 3 females in right side, and 2 subject in left side experienced the symptoms. 6 males and 2 females in right side while 10 males and 4 females in left side had none of the above mentioned symptoms.

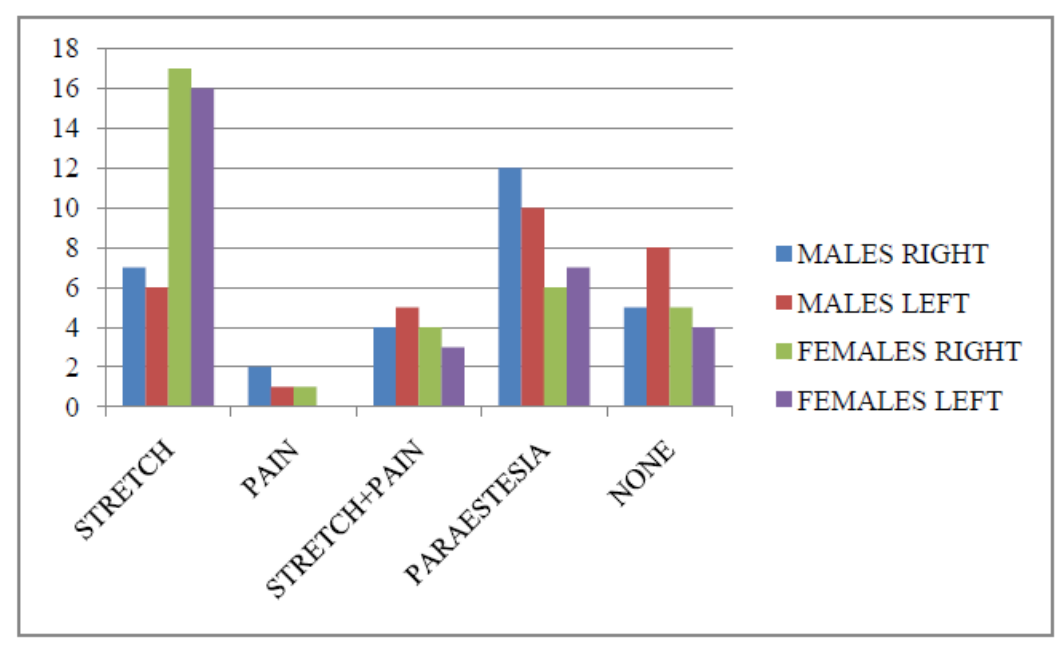

Figure3. Showing results for ULTT 3 Ulnar Nerve bias

Table3. Ulnar nerve bias

\begin{tabular}{|l|l|l|l|l|}
\hline \multirow{2}{*}{ Parameters } & Males & \multicolumn{2}{l|}{ Females } \\
\cline { 2 - 5 } & Right & Left & Right & Left \\
\hline Stretch & 7 & 6 & 17 & 16 \\
\hline Pain & 2 & 1 & 1 & 0 \\
\hline Stretch+Pain & 4 & 5 & 4 & 3 \\
\hline Paraestesia & 12 & 10 & 6 & 7 \\
\hline None & 5 & 8 & 5 & 4 \\
\hline
\end{tabular}

From figure 3,In case of ULTT 3, out of 60 candidates,

Stretch was reported by 7 males in right upper extrimity ,16 males in left upper extrimity and 17 females in right upper extrimity , 16 females in left upper extremity. 2 males and 1 female in right side of upper limb while 1 male in left side of upper limb reported pain .stretch plus pain was experienced by 4 males in right side 5 males in left side and 1 female in right side, 3 in left side .in case of paresthesia 12 males in right side and 10 males in left side. 6 females in right side and 7 females in left side experienced the symptoms. 5 males in right side and 7 males in left side, 5 females in right side and 4 females in left side of upper extrimity had none of the above mentioned symptoms.

Using unpaired $t$ test data is analysed.significant $p<0.05$ for pain and paresthesia symptom in upper limb tension test for maedian nerve. For upper limb tension test, radial nerve bias significant $p<0.05$ for pain. In case of upper limb tension test ulnar nerve bias, $\mathrm{p}<0.05$ for stretch and paresthesia.

\section{DisCuSSION}

This study was conducted to find out upper limb neural tissue extensibility in computer operators who are asymptomatic in relation to upper quadrant involvement. The positive neural tissue tension test in 
most of the subjects came to positive results. But a positive tension test does not necessarily indicate a mechanical or a physiological disorder.

Total 60 candidates who participated in the study used chairs with adjustable backrest while working on their PCs. But $80 \%$ had no idea about the ergonomic seating posture and were unable to maintain the ideal posture comprising the neck and shoulder component throughout the working hours. So lesser risk of developing problem in that participants who have an idea about ergonomic seating posture during use of computer.

In case of upper limb tension test1, Median nerve bias, stretch felt was more in females than in males, pain was minimal, and paresthesia was the experienced by both the genders in bilateral upper limbs. The component of parasthesia was severe as compared to that of pain and stretch in both the upper extremities.

In upper limb tension test 3, Radial nerve bias most of the male and female candidates reported only stretch, bilaterally. Out of 30 males, 6 reported no feeling of pain, stretch or parasthesia bilaterally for radial nerve while other 4 reported to have felt nothing on left side felt specifically.

In case of upper limb tension test 3, ulnar nerve bias, female candidate perceived more stretch bilaterally than male counterparts. Only two candidates reported pain. Paresthesia was experienced by 12 male candidates in dominant upper limb and 10 male candidates in left upper limb. Out of female candidates, 6 females perceived parasthesia in right and 7 in non dominant upper limb. Nothing was felt by 5 candidates of both genders bilaterally.

Thus from above observed values we could say that median nerve is more sensitive towards stretch.

The resultant positive median nerve bias test may be due to anatomy of brachial plexus.

pathomechanics may lead to a pathophysiological situation separately or in combination with abnormal movement mechanics $[14,16]$.

A force is placed on a surrounding symptomatic interfacing structure on application of provocation test, keeping the system normal in itself. Compression of shorter duration of intact peripheral nerves or nerve roots has been stated to cause paraesthesia rather than pain as a result of ischemia. Parasthesia is not through mechanical deformation of nerve fibre[16,18,19]. Hamouda M. et al reviewed that the median nerve biased neural tissue provocation test as a useful diagnostic test for assessment of cervicobrachial pain disorders on the basis of anatomical and clinical evidence [5].

If adhesions hinder physiological movement and the even distribution of tension in the nerve, intraneural microcirculation could be impaired. However, nerve function as well as communication and nutritional transport systems "antegrade and retrograde transport" depend on an adequate supply of oxygen to the nerve fibres [10,11].

Scapular position and mobility influenced by the crvico-thoracic posture. This is demonstrated to be associated with subacromial conflict and gleno-humeral instability; resultant Shoulder protraction can cause neurovascular impingement having an impact on median nerve function thus increasing the risk of development of upper quadrant pain [21,22].

Posture and limb movements may lead to alter the physical stresses on the nerve trunk which may results in modification of the structural and biomechanical properties of the same nerve. Physical stress is defined as the "force or load acting on a given area of tissue" $[7,8,9]$. Mueller and Maluf in their Physical Stress Theory stated that the adaptive response of the biological tissue will depend on the physical stress applied on that particular tissue [9]. The three concepts elaborated regarding neural tissue, for adaptations to any mechanical stress, according to the force applied are, if applied force is more than necessary, tissue responds by excessive elongation, if force is adequate the neural tissue shows no adaptation, and if the force is less than the tissue response by decreased ability to tolerate the stress. This occurs due to the plasticity of the neural tissue that adapts itself to the applied functional demands $[5,6,8,9]$. Neural tissue adapts to normal joint motion through a gliding mechanism that allows movement with minimal or no strain on the individual nerve fibres. Also the nerve trunk stretches to about $6-8 \%$ of its original length, which makes it relatively resistant to mechanical load as compare the nerve root. Micro vascular circulation is interfered with increased elongation and compression which may lead to perineuraloedema, fibrosis, and degeneration of nerve fibres. Alterations in microcirculation and tissue pressures around the nervous systems are likely to be present well before the structural changes are evident $[2,3,6,8,9]$. 


\section{CONClusion}

This study indicates the susceptibility of the median nerve and ulnar nerve towards posture related reduced extensibility. Thus, abnormal posture leads to abnormal neural tissue mobility. Hence stretching the neural tissue at appropriate age may be precautionary for future problems.

\section{REFERENCES}

[1] Sunderland S (1990) the anatomy and physiology of nerve injury. Muscle and nerve;13;771-784

[2] Sunderland S, Bradley KC (1961a) Stress-strain phenomena in human peripheral nerve trunks.brain;84;102-124

[3] Topp KS, Boyd BS. Structure and biomechanics of peripheral nerves: nerve responses to physical stresses and implications for physical therapist practice. Physical Therapy journal. 2006; 86:92-109.

[4] Levangie P.; NorkinC.; Joint Structure and Function:A Comprehensive Analysis;4;13;503-506

[5] Hamouda M.; The Neural Tissue Provocation Test as A Diagnostic Tool In The Assessment of Cervicobrachial Pain Disorders:A Critical Appraisal;University of Wales;July 2003.

[6] Butler DS . Mobilization of the nervous system. New York :Churchill Livingstone 1991

[7] Das S.;Prevalance Of Adverse Neural Tension In Complaints Of Neck, Arm And Shoulder (Cans) Among Computer Operators; Rajiv Gandhi University Of Health Sciences Bangalore, Karnataka

[8] Shacklock M.;Neurodynamics; journal of Physiotherapy;jan1995;1;1-13

[9] Mueller M, Maluf K. Tissue adaptation to physical stress: a proposed "physical stress theory" to guide physical therapist practice, education,and research. PhysTher. 2002;82:383- 403.

[10] Ommaya AK. Mechanical properties of tissues of the nervous system. Journal of Biomechanics. 1968 Jul 1;1(2):127IN23137-36138.

[11] Ikada Y. Challenges in tissue engineering. Journal of the Royal Society Interface. 2006 Oct 22;3(10):589-601.

[12] Gilbert KK, Smith MP, Sobczak S, James CR, Sizer PS, Brismée JM. The Journal of Manual \& Manipulative Therapy. 2015/12/01 00:00; 23(5)239-245

[13] Radhakrishnan K, Litchy WJ, O'Fallon WM, Kurland LT. Epidemic way of cervical radiculopathy. A population-based study from Rochester, Minnesota 1976 through 1990. Brain 1994;117: 325-335

[14] Cote P, Cassidy D, Carroll L.; The factors associated with neck pain and its related disability in the Saskatchewan population. Spine (2000); 25: 1109-17.

[15] Cote P, Cassidy J, Carroll L. The Saskatchewan health and back pain survey. The prevalence of neck pain and associated disability in Saskatchewan adults. Spine 1998; 23: 1689-98.

[16] Caplan L. Management of cervical radiculopathy. Eur Neurol. 1995;35:309-320.

[17] Gerard A Malanga, MD, Michael A Romello, MD, Cervical Radiculopathy e medicine article March 2008(2)

[18] Heckmann J, Lang J, Zobelein I, et al. Herniated cervical intervertebral discs with radiculopathy: an outcome study of conservatively or surgically treated patients. J Spinal Disord. 1999;12:396401

[19] Y Tanaka, S.Kukbun, T Sato. Cervical radiculopathy and its unsolved problems. Current orthopedics (1998) 12, 1-6

[20] Weiderholt WC. Neurology for non-neurologists. 4th edition. Philadelphia: WB. Sauders Co; 2000. P 205-11

[21] Elvey RL .Painful restriction of shoulder movement: a clinical observational study. In: Proceedings ,Disorders of the knee ankle and shoulder.1979.Western Australia institute of Technology, Perth

[22] Elvey RL .Treatment of arm pain associated with abnormal brachial plexus tension . Australian Journal of Physiotherapy 1986;32:224-229 